\title{
Diverticulitis de la flexura hepática del colon*
}

\author{
Drs. ALEJANDRO PUGLIESE ${ }^{1}$, GERMÁN VISCIDO ${ }^{1}$, HÉCTOR PICÓN-MOLINA ${ }^{1}$, \\ ALEJANDRO DONIQUIAN ${ }^{1}$, RAFAEL PALENCIA ${ }^{1}$. \\ 1 Servicio de Cirugía General, Clínica Universitaria Reina Fabiola. \\ Córdoba, Argentina.
}

Abstract

\section{Colonic hepatic flexure diverticulitis}

Objective: Right colon diverticulitis is a rare entity in our means. Frequently, this entity is mistaken for other abdominal conditions, usually appendicitis, right colon cancer or acute cholecystitis. We found no specific reports about their location in the hepatic flexure of the colon. Material and Method: 58-years-old male who consulted the emergency department with abdominal pain and palpable mass in right upper quadrant. Abdominal CT revealed diverticulitis of the hepatic flexure of the colon. Results: Successful outcome with medical treatment. The patient is asymptomatic at 3 months of follow. Conclusion: Diverticulitis of the hepatic flexure of the colon is a rare entity that can mimic other diseases and usually responds to conservative treatment.

Key words: Diverticulitis, right colon, medical treatment.

\section{Resumen}

Introducción: La diverticulitis del colon derecho es una entidad poco frecuente en nuestro medio. Con relativa frecuencia, esta entidad es confundida con otros procesos abdominales, habitualmente apendicitis, cáncer de colon derecho o colecistitis aguda. No encontramos reportes específicos sobre su localización en la flexura hepática del colon. Material y Método: Varón 58 años quien consultó en urgencias por dolor abdominal y masa palpable en hipocondrio derecho. Se realiza TC abdominal que revela diverticulitis del ángulo hepático del colon. Resultados: buena evolución con tratamiento médico; el paciente se encuentra asintomático a tres meses del alta. Conclusión: La diverticulitis del ángulo hepático del colon es una entidad poco habitual, que puede simular otras patologías y que habitualmente responde al tratamiento conservador.

Palabras clave: Diverticulitis, colon derecho, tratamiento médico.

*Recibido el 12 de junio de 2012 y aceptado para publicación el 17 de julio de 2012.

Los autores no refieren conflictos de interés.

Correspondencia: Dr. Germán Viscido

Oncativo 1240, Córdoba, Argentina.

germanviscido@gmail.com 


\section{Introducción}

Los divertículos del colon derecho son infrecuentes en Occidente. Afectan al 5\% de la población adulta y dan cuenta del $1,5 \%$ de los pacientes con diverticulitis colónica. Su prevalencia es más elevada en la población asiática, donde representa el 20\% de la enfermedad diverticular y el $75 \%$ de los casos de diverticulitis ${ }^{1,2}$.

Con frecuencia es diagnosticada de forma errónea y hallada incidentalmente en el momento de la cirugía por una presunta apendicitis aguda ${ }^{3}$.

\section{Descripción del caso}

Paciente masculino de 58 años de edad sin antecedentes patológicos. Consulta en emergencias por dolor abdominal en hipocondrio derecho de $24 \mathrm{~h}$ de evolución, de comienzo gradual, de carácter continuo con exacerbaciones cólicas y sin irradiación; al inicio de intensidad moderada aumentando a severa con la evolución. El dolor no presentó relación con la ingesta y disminuyó parcialmente con la ingesta de antiespasmódicos. No tuvo episodios de dolor previo de similares características. Tampoco ictericia, coluria o prurito; náuseas, vómitos, fiebre, síntomas urinarios, alteración en las características de sus deposiciones o en su hábito intestinal.

Se presenta en buenas condiciones generales, con hemodinamia y signos vitales normales. Al examen físico se observa un abdomen globuloso, levemente distendido y simétrico; se palpa tenso, con dolor a la palpación superficial y profunda en hipocondrio y flanco derechos con francos signos de irritación peritoneal en dicha zona, palpándose además una tumoración de 10 centímetros de diámetro, redondeada, consistencia dura, dolorosa y que incursiona con los movimientos respiratorios. La auscultación es de características normales. El resto del examen físico sin particularidades. Laboratorio al ingreso se objetiva una leve leucocitosis (Tabla 1).

Con diagnóstico presuntivo de Colecistitis Aguda se realiza ecografía abdominal que visualiza vesícula biliar con barro biliar sin signos inflamatorios (Figura 1). Luego de hospitalizar al paciente y ante los

\section{Tabla 1. Exámenes de laboratorio al ingreso}

\begin{tabular}{|ll|}
\hline $07 / 09 / 2010$ & $15,2 \mathrm{~g} \%$ \\
\hline Hemoglobina & $45,3 \%$ \\
\hline Hematocrito & $12.200 / \mathrm{mm}^{3}$ \\
\hline Leucocitos & $\mathrm{NS} 75 \% / \mathrm{L} 22 \% / \mathrm{B} \%$ \\
\hline & $13 \mathrm{~mm} / \mathrm{h}$ \\
\hline VSG & $0,37 \mathrm{~g} / 1$ \\
\hline Urea & $0,78 \mathrm{mg} \%$ \\
\hline Creatinina & $35 \mathrm{U} / \mathrm{I}$ \\
\hline Amilasa & $13 \mathrm{U} / \mathrm{L}$ \\
\hline GOT & $37 \mathrm{U} / \mathrm{L}$ \\
\hline GPT & $1,17 \mathrm{mg} \%$ \\
\hline Bilirrubina T & $0,52 \mathrm{mg} \%$ \\
\hline Bilirrubina D & 1 eve $\mathrm{piuria}$ \\
\hline Orina completa & \\
\hline
\end{tabular}
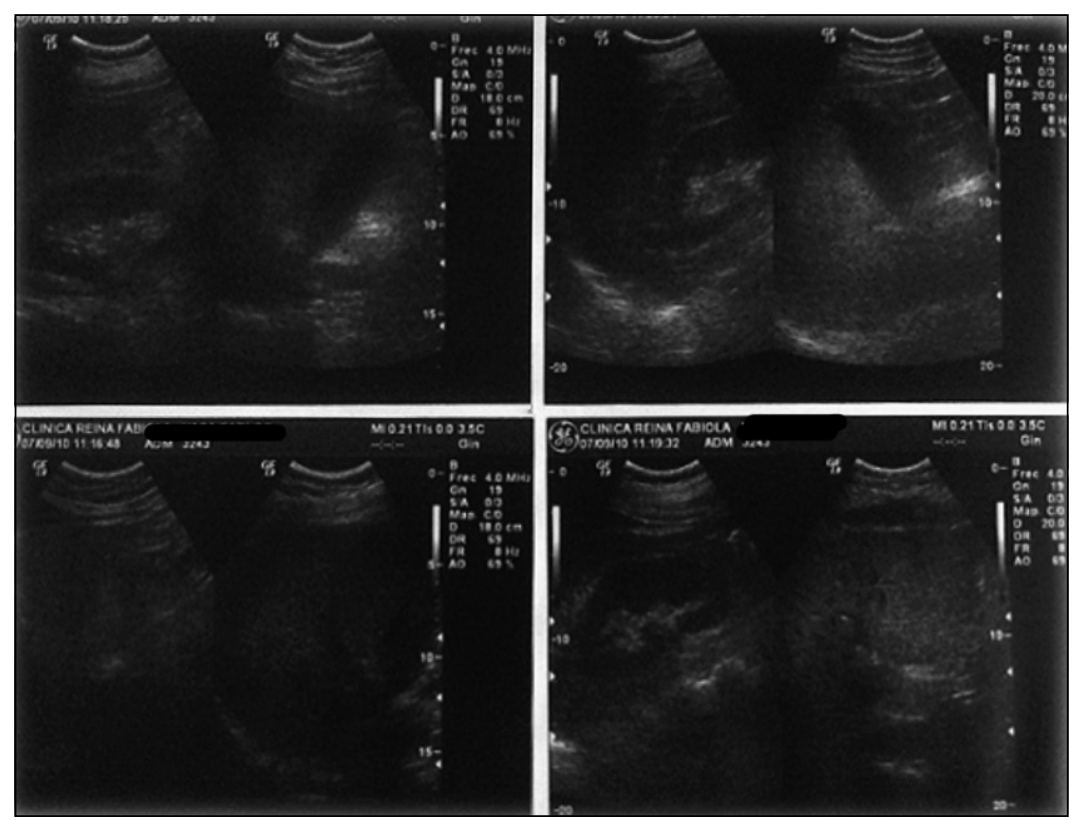

Figura 1. Ecografía abdominal. 

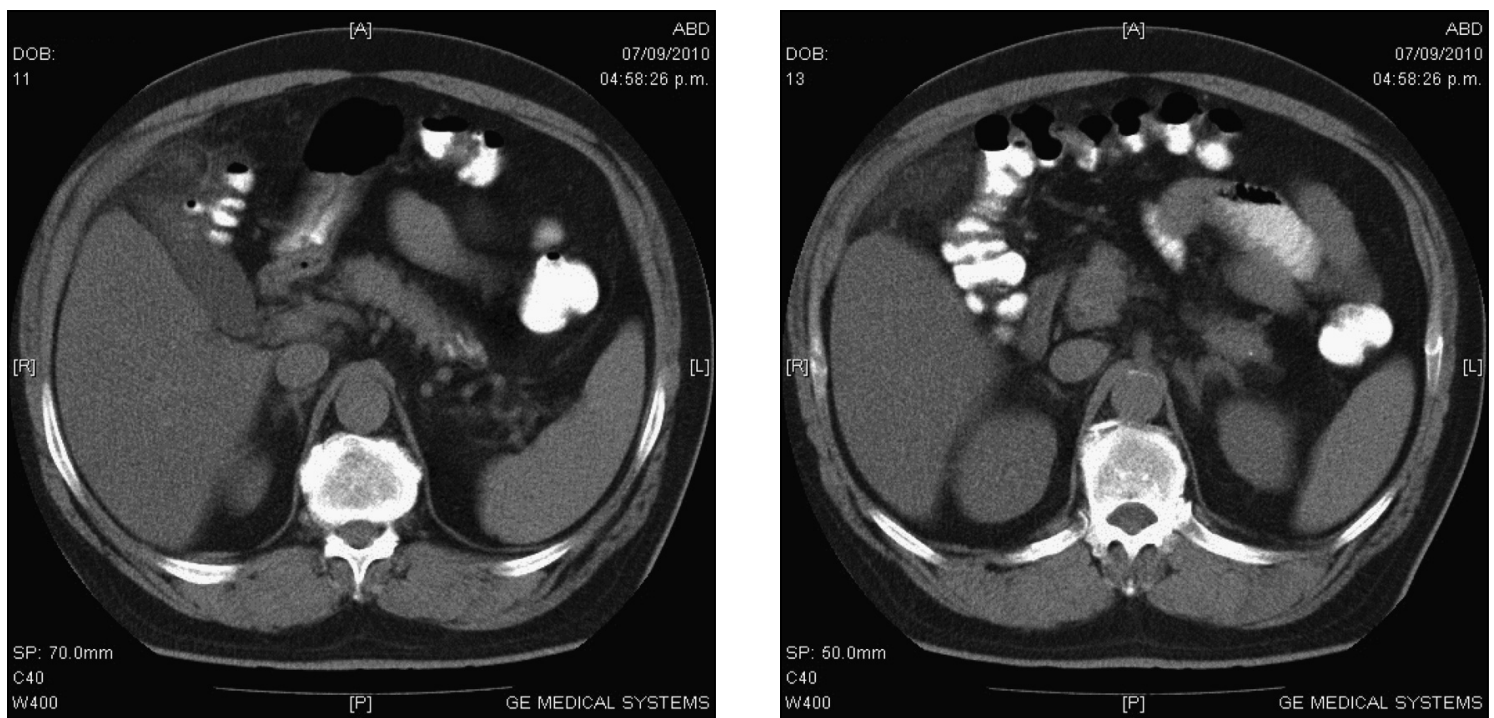

Figuras 2 y 3. El TC muestra un severo proceso inflamatorio de origen diverticular a nivel del ángulo hepático del colon con engrosamiento parietal y enrarecimiento de la grasa circundante.

hallazgos en el examen físico del abdomen no coincidentes con los hallazgos ecográficos se realiza un scanner abdominal con doble contraste con el diagnóstico de tumor inflamatorio del hipocondrio derecho. El TC muestra un severo proceso inflamatorio de origen diverticular a nivel del ángulo hepático del colon con engrosamiento parietal y enrarecimiento de la grasa circundante (Figuras 2 y 3 ).

Realizado el diagnóstico etiológico de diverticulitis de la flexura hepática del colon, en un paciente con buenas condiciones generales, sin signos de respuesta inflamatoria sistémica y sin complicaciones locales de la diverticulitis, se decide inicialmente manejo conservador con reposo intestinal, analgésicos y antibióticos. Presenta buena evolución, otorgándole el alta hospitalaria al $4^{\circ}$ día.

A los 3 meses del episodio, el paciente se encuentra en buenas condiciones, asintomático y se realizó una videoendoscopia baja que muestra diverticulosis en colon sigmoides y algunos en el ángulo hepático sin signos de estenosis.

\section{Discusión}

Los divertículos colónicos son herniaciones de la mucosa que se proyectan a través de aberturas creadas por los vasos rectos al penetrar en la pared ${ }^{4}$.

Cuando son solitarios, usualmente son congénitos y "divertículos verdaderos", encontrándose habitualmente en el ciego o en las proximidades de la válvula íleo-cecal ${ }^{5}$.
Se presenta a una edad más temprana, en promedio a los 35 a 45 años de edad, sin tener diferencias en la distribución respecto al sexo ${ }^{2,4}$.

La diverticulitis del colon derecho (DCD) frecuentemente es diagnosticada de forma errónea como apendicitis aguda, pudiendo además, simular otras enfermedades como el cáncer de colon derecho, colecistitis o enfermedad úlcero-péptica ${ }^{3,6}$. La frecuencia reportada de esta enfermedad varía desde 1:34 a 1:300 apendicectomías?.

Se presenta habitualmente con dolor abdominal derecho o generalizado, en la mayoría de los reportes con 2 días o más de evolución, sin la migración típica desde el epigastrio como en la apendicitis, y con baja frecuencia de síntomas y signos generales con náuseas, vómitos, anorexia, fiebre o leucocitosis $^{7}$. En nuestro caso la presentación clínica asociada a la palpación de una tumoración en el hipocondrio derecho llevó a un diagnóstico presuntivo de una Colecistitis Aguda.

Se ha reportado que el diagnóstico preoperatorio correcto ocurre en el 4 al $16 \%$ de los casos ${ }^{8}$. Los métodos por imágenes son fundamentales para el diagnóstico correcto en el prequirúrgico, siendo la tomografía de abdomen el estudio que presenta mayor sensibilidad o especificidad ${ }^{9}$. Los criterios tomográficos para el diagnóstico de DCD incluyen engrosamiento de la pared del colon, infiltración de la grasa pericolónica y absceso pericolónico o a distancia ${ }^{9,10}$.

Realizado el diagnóstico preoperatorio de DCD no complicada el tratamiento es conservador. Se 
ha comunicado una mejor respuesta al tratamiento médico que en diverticulitis del colon izquierdo y que la historia natural es benigna a pesar de su evolución ${ }^{7,9,11}$. La recurrencia oscila entre el $10 \%$ y el $25 \%$ durante seguimientos a 10 años y no se han comunicado complicaciones severas durante las mismas $^{12,13}$.

El tratamiento quirúrgico debe adecuarse a la extensión del proceso inflamatorio y las complicaciones. Si durante la cirugía se evidencia leve inflamación del colon no se recomienda tomar ninguna conducta más que una apendicectomía profiláctica, debiendo realizarse posteriormente un estudio del colon mediante colonoscopia o colon por enema ${ }^{7}$. La diverticulectomía es el procedimiento menos extenso que se puede realizar ${ }^{12}$. La discrepancia en cuanto a la estrategia de tratamiento ante el hallazgo de una DCD se debe a que la historia natural es poco conocida.

La hemicolectomía derecha, técnica con baja morbi-mortalidad, debe ser realizada de acuerdo a las preferencias del paciente o en caso de sospecha de malignidad ${ }^{4,5,7}$.

La diverticulitis complicada que presenta un absceso debe ser tratada con drenaje percutáneo del absceso como primer enfoque terapéutico o mediante cirugía ${ }^{8}$.

\section{Conclusión}

La diverticulitis del colon derecho es una patología poco frecuente en nuestro ámbito. Sigue siendo frecuentemente diagnosticada, a pesar de los avances en los estudios imagenológicos, durante una presunta apendicectomía, no existiendo consenso en la literatura sobre cuál es la mejor conducta terapéutica durante su hallazgo en cirugía. De establecer el diagnóstico preoperatorio se debe instaurar tratamiento médico en casos no complicados, presentando habitualmente una buena evolución, como en nuestro caso.

\section{Referencias}

1. Hughes L. Postmortem survey of diverticular disease of the colon. I. Diverticulosis and diverticulitis. Gut 1969;10:336-44.

2. Scatarige J, Fishman E, Crist D, Cameron J, Siegelman S. Diverticulitis of the right colon: CT observations. AJR Am J Roentgenol. 1987;148:737-9.

3. Nirula R, Greaney G. Right-sided diverticulitis: a difficult diagnosis. Am Surg. 1997;63:871-3.

4. Fischer M, Farkas A. Diverticulitis of the cecum and ascending colon. Dis Colon Rectum 1984;27:454-8.

5. Telem D, Buch K, Nguyen S, Chin E, Weber K, Divino C. Current Recommendations an Diagnosis and Management of Right-Sided diverticulitis. Gastroenterol Res Pract. 2009;359485. Epub 2009 Mar 24.

6. Sugihara K, Muto T, Morioka Y, Asano A, Yamamoto T. Diverticular disease of the colon in Japan. A review of 615 cases. Dis Colon Rectum 1984;27:531-7.

7. Yang H, Huang H, Wang Y, Hsieh C, Cheng P, Jeng L, et al. Management of right colon diverticulitis: a 10year experience. World J Surg. 2006;30:1929-34.

8. Li-rung S, Shee-Chan L, Shou-Chuan S, Chin-Roa K, Sun-Yen C. Decision making in right-sided diverticulitis. World J Gastroenterol. 2003;9:606-8.

9. Oudenhoven L, Koumans R, Puylaert J. Right colonic diverticulitis: US and CT findings - new insights about frequency and natural history. Radiology 1998;208:6118.

10. Chou Y, Lai B, Hsu C. Sonographic appearances of right-sided colonic diverticulitis. JMEUE 1996;4:180-3.

11. Ngoi S, Chia J, Goh M, Sim E, Rauff A. Surgical management of right colon diverticulitis. Dis Colon Rectum 1992;35:799-802.

12. Komuta K, Yamanaka S, Okada K, Kamohara Y, Ueda $\mathrm{T}$, Makimoto $\mathrm{N}$, et al. Toward therapeutic guidelines for patients with acute right colonic diverticulitis. Am J Surg. 2004;187:233-7.

13. Fang J, Chen R, Lin B, Hsu Y, Kao J, Chen M. Aggressive resection is indicated for cecal diverticulitis. Am J Surg. 2003;185:135-40. 